\title{
Avances en corteza auditiva
}

\author{
Advances in auditory cortex
}

\author{
Natalia Jara $0^{1,2}$, Paul H. Délano $\mathbf{R}^{2,3}$.
}

\begin{abstract}
RESUMEN
El sistema auditivo nos permite detectar e interpretar las señales acústicas del medio ambiente y así modificar nuestro comportamiento. En humanos la corteza auditiva se ubica en el giro temporal superior del lóbulo temporal. Esta corteza presenta una organización estructural y funcional característica, que se ha identificado en muchas especies de mamíferos. Las áreas de organización de la corteza auditiva son: (i) una región central denominada corteza auditiva primaria o core, que corresponde al primer nivel de procesamiento, cuyas características cito-arquitectónicas y funcionales principales son poseer una capa IV prominente y presentar una organización tonotópica especular. Además, (ii) una región circundante conocida como cinturón o belt, que corresponde a las cortezas secundarias que participan de la localización espacial y reconocimiento del sonido, como también en el procesamiento del habla. Por último, (iii) las áreas de asociación auditiva integran la información auditiva con la de otros sistemas sensoriales. En este artículo se revisan las bases neuroanatómicas y las propiedades funcionales de la corteza auditiva, las que constituyen pilares fundamentales para el desarrollo de métodos diagnósticos y terapéuticos del procesamiento auditivo central.
\end{abstract}

Palabras claves: Sistema auditivo central, corteza auditiva, organización tonotópica, procesamiento auditivo central.

\begin{abstract}
The auditory system allows us to detect and interpret the acoustic signals of the environment and thus change our behavior. In humans, the auditory cortex is located in the superior temporal gyrus of the temporal lobe. This cortex has a characteristic structural organization and functionality that have been identified in many mammalian species. The auditory cortex has different organizational areas: (i) a core called "primary auditory cortex," which corresponds to the first level of processing, and its cyto-architectural and physiological main features are to present a prominent layer IV and to display a

1 Programa de Magíster en Morfología, Escuela de Posgrado, Facultad de Medicina, Universidad de Chile.

2 Laboratorio Neurobiología de la Audición, Programa de Fisiología y Biofísica, ICBM, Facultad de Medicina, Universidad de Chile.
\end{abstract}

3 Departamento de Otorrinolaringología, Hospital Clínico de la Universidad de Chile.

Recibido el 30 de junio, 2014. Aceptado el 3 de agosto, 2014. 
mirror-tonotopic organization. In addition, (ii) a surrounding region known as belt that corresponds to the secondary auditory cortices and participates in the location and recognition of sound, as well as in speech processing. Finally, (iii) auditory association areas that integrate auditory information with other sensory systems. In this article, the neuroanatomical bases and functional properties of auditory cortex processing are reviewed. These topics constitute the foundations for the development of diagnostic tools and therapeutic procedures of central auditory processing.

Key words: Central auditory system, auditory cortex, tonotopic organization, central auditory processing.

\section{INTRODUCCIÓN}

Si bien la cóclea es fundamental para la detección de las ondas acústicas que nos rodean, es en la corteza auditiva donde se crea la percepción del sonido y así se modifica nuestro comportamiento ${ }^{1}$. El sistema auditivo central y periférico nos permite detectar y discriminar amplios rangos de las características físicas del sonido, que en el ser humano va de 20 a $20.000 \mathrm{~Hz}$ en frecuencia, y de 0 a 140 dB SPL en intensidad. Además, el sistema auditivo debe codificar estímulos acústicos complejos como los que se producen durante una conversación, una sinfonía, o al distinguir el llanto de un bebé del ruido de fondo. Estas habilidades están determinadas tanto por el receptor periférico, la cóclea, como por el sistema auditivo central, incluyendo los núcleos subcorticales de la vía auditiva y la corteza auditiva ${ }^{2-3}$. En este artículo se revisan conceptos anatómicos y funcionales de las vías auditivas centrales y de la corteza auditiva.

\section{DE LA CÓCLEA A LA CORTEZA: VÍA AUDITIVA CENTRAL}

La cóclea se localiza en la cápsula ótica del hueso temporal de los distintos mamíferos y se caracteriza por estar compuesta de tres rampas: vestibular, timpánica y media. Las rampas vestibular y timpánica contienen perilinfa, mientras que la rampa media, endolinfa. En la rampa media se ubica el órgano de Corti que corresponde al receptor periférico del sistema auditivo. Este se apoya sobre la membrana basilar y posee a las células ciliadas internas y externas, responsables de la mecano-transducción auditiva ${ }^{4}$.
Las células ciliadas internas son inervadas por fibras aferentes del nervio auditivo cuyos axones se dirigen al núcleo coclear. Según la especie estudiada, se describe que cada célula ciliada interna recibe 10 a 20 sinapsis de fibras aferentes tipo I del nervio auditivo $0^{5}$. Las fibras del nervio auditivo ingresan al núcleo coclear y se dividen en "Y", inervando a la región ventral y dorsal de este núcleo. A su vez, el núcleo coclear se compone de una región ántero-ventral, póstero-ventral y dorsal, desde donde emergen las estrías acústicas ventral, intermedia y dorsal respectivamente. Las estrías acústicas ventral e intermedia se dirigen al complejo olivar superior, mientras que la dorsal va directamente al colículo inferior ${ }^{6}$. Las vías auditivas del tronco encefálico, provenientes del complejo olivar y del lemnisco lateral convergen a nivel de los colículos inferiores en el mesencéfalo. Los colículos inferiores poseen un núcleo central organizado en láminas de isofrecuencia, que se ordenan en forma tonotópica: las láminas superficiales poseen neuronas de frecuencias características bajas y las profundas de frecuencias altas. Además del núcleo central de los colículos inferiores, éstos presentan una corteza dorsal que recibe principalmente eferencias y proyecciones desde otras zonas del cerebro? El núcleo central del colículo inferior proyecta hacia la porción ventral del cuerpo geniculado medial del tálamo, y a su vez las fibras talámicas que se dirigen a la corteza auditiva primaria se originan en la región ventral del cuerpo geniculado medial ${ }^{8}$. Una de las propiedades fundamentales de los núcleos de la vía auditiva central es que presentan tonotopia 0 un orden anatómico según la frecuencia característica de sus neuronas (Figura 1). 


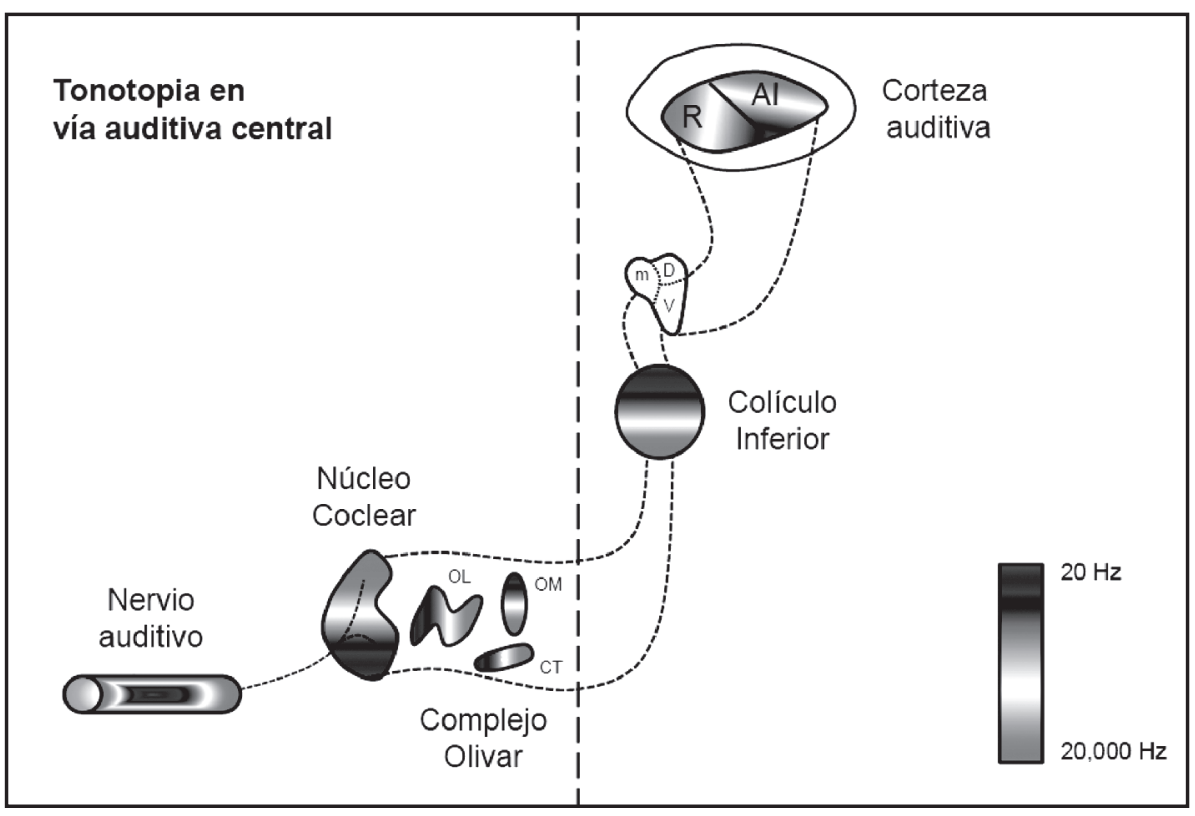

Figura 1. Esquema de la vía auditiva central. Se muestran los diferentes núcleos de la vía aferente del sistema auditivo central junto con su tonotopía. Las conexiones ascendentes desde el nervio auditivo hasta la corteza auditiva se muestran en líneas segmentadas. El orden tonotópico está representado en escala de colores, donde las frecuencias bajas (graves) están representadas en azul y las frecuencias altas (agudos) en rojo. A nivel del complejo olivar superior se produce el primer cruce de la vía auditiva. Se muestran los diferentes núcleos olivares, OL: olivar lateral OM: olivar medial CT: cuerpo trapezoide. El cuerpo geniculado medial con sus tres regiones m: medial, V: ventral y D: dorsal. La corteza auditiva posee una tonotopía especular entre sus regiones rostral: $\mathrm{R}$ y corteza auditiva primaria: $\mathrm{Al}$.

\section{CORTEZA CEREBRAL DE MAMÍFEROS}

La corteza cerebral o neocorteza de mamíferos está organizada en seis capas (denominadas del I al VI desde superficial a profundo), constituidas por variados tipos de neuronas que poseen diferentes propiedades intrínsecas y de conectividad hacia otras estructuras del sistema nervios $0^{9}$. La capa I 0 "lámina molecular" está constituida por dendritas apicales de neuronas cuyos cuerpos celulares están en la capa $\mathrm{V}$ y $\mathrm{VI}$, axones provenientes de los hemisferios cerebrales e interneuronas con orientación horizontal, Ilamadas células de CajalRetzius. Funcionalmente se postula que la capa I se ha asociado con la integración sináptica de la corteza $^{10}$. La capa II o "lámina granular externa" está constituida por neuronas de tamaño mediano y pequeño junto con interneuronas, mientras que la capa III o "lámina piramidal externa" está conformada por neuronas piramidales medianas. Funcionalmente, las capas II y III participan de la comunicación intracortical entre neuronas, tanto entre regiones ipsilaterales como contralaterales. La capa IV o “lámina granular interna" está formada por interneuronas excitatorias, llamadas células estrelladas, y recibe aferencias talámicas específicas. Esta capa cortical es fundamental en las cortezas sensoriales primarias, como la corteza auditiva, debido a que reciben las proyecciones de las divisiones sensoriales del tálamo. La capa V o "lámina piramidal interna" contiene grandes neuronas piramidales asociadas a grupos específicos de interneuronas. Las neuronas de esta capa proyectan a estructuras subcorticales, como el cuerpo estriado, tronco cerebral y médula espinal. La capa VI o "lámina multiforme" está constituida por neuronas piramidales modificadas, las cuales forman los circuitos córtico-talámicocorticales. Específicamente, en la corteza auditiva las neuronas piramidales de la capa $\mathrm{V}$ y $\mathrm{VI}$ son el origen del sistema eferente córticoolivococlear ${ }^{10,11}$. 


\section{CORTEZA AUDITIVA}

La corteza auditiva puede ser clasificada en primaria o secundaria, dependiendo de las proyecciones talámicas que reciba y de sus propiedades electrofisiológicas. La corteza auditiva primaria, conocida también como core, recibe proyecciones directas de la porción ventral del cuerpo geniculado medial y posee neuronas que responden con latencias cortas y que tienen alta especificidad por la frecuencia de los estímulos. Por otra parte las cortezas secundarias, conocidas como belt, reciben proyecciones de la porción dorsal y medial del cuerpo geniculado medial y tienen latencias más largas y poseen una menor especificidad frecuencial ${ }^{12}$. Además de las cortezas auditivas del core y belt existen áreas auditivas de asociación 0 parabelt que integran la información auditiva con la de otros sistemas sensoriales ${ }^{13}$.

\section{Corteza auditiva en humanos}

Muchos investigadores han descrito la organización de la corteza auditiva humana, pero fue en 1909 que el investigador alemán Korbinian Brodmann ${ }^{14}$ publicó el principal estudio de la citoarquitectura de la corteza cerebral, a través del cual describió diversas áreas cerebrales que desde entonces se denominan con números y que se conocen como las áreas de Brodmann (BA) ${ }^{15}$. Según esta nomenclatura, se describen cuatro regiones auditivas (áreas 22, 41, 42 y 52 de Brodmann) ubicadas en el lóbulo temporal específicamente en el giro temporal superior donde se forma el giro de Heschl, lugar de ubicación de la corteza auditiva ${ }^{16}$.

La corteza auditiva primaria en humanos correspondería a las áreas 41 y 42 de Brodmann ${ }^{17}$. Morosan y cols $^{18}$ subdividen en 4 regiones al área 41 de Brodmann (corteza auditiva primaria): Te 1.0, 1.1, 1.2 y 1.3, mientras que el área 42 de Brodmann corresponde a Te2 y la subdividen en las regiones Te 2.1 y Te 2.2. Por otra parte los autores catalogan a las áreas 22 y 52 de Brodmann como cortezas secundarias y las denominan como Te3 y Tel respectivamente. Sin embargo, aún existe discrepancia de los bordes citoarquitectónicos precisos de cada área auditiva, por lo que se requieren más estudios para poder comprender la relación entre estructura y función de la corteza auditiva ${ }^{19}$. La representación de las áreas anteriormente descrita se muestra en la Tabla 1.

\section{Citoarquitectura de la corteza auditiva}

La principal característica citoarquitectónica de la corteza auditiva primaria (Al) es presentar las capas II y IV densas y uniformes. La capa granular interna (capa IV)

\section{Tabla 1. Nomenclatura de corteza auditiva humana}

\begin{tabular}{lllll}
\hline Investigador & Giro de Heschl & Plano Temporal & $\begin{array}{l}\text { Giro Temporal } \\
\text { superior lateral }\end{array}$ & \multicolumn{1}{c}{ Plano Polar } \\
\hline $\begin{array}{l}\text { Brodmann, } \\
1909\end{array}$ & 41 & 42 & 22 & 52 \\
\hline $\begin{array}{l}\text { Von Economo y } \\
\text { Koskinas, 1925 }\end{array}$ & TC/TD & TB & TA & IBT \\
$\begin{array}{l}\text { Galaburda y } \\
\text { Sanides, 1980 }\end{array}$ & Kam/ Kalt & $\begin{array}{l}\text { PaAi, PaAe, } \\
\text { PaAc, Tpt }\end{array}$ & PaAe, Tpt & ProA \\
$\begin{array}{l}\text { Rivier y Clarke, } \\
1997\end{array}$ & Al & LA/PA & STA & MA \\
$\begin{array}{l}\text { Morosan y col, } \\
2005\end{array}$ & $\begin{array}{l}\text { Te1 } \\
\text { (Te 1.1/1.0, 1.2) }\end{array}$ & $\begin{array}{l}\text { Te2 } \\
\text { (Te2.1/Te2.2) }\end{array}$ & Te3 & TI \\
\hline
\end{tabular}

Modificado de Galaburda y Sanides, 1980. Cytoarchitectonic organization of the human auditory cortex. J Comp Neurol 190: 597-610. Modificado de Rivier y Clarke, 1997. Cytochrome oxidase, acetylcholinesterase, and NADPH-diaphorasestaining in human supratemporal and insular cortex: evidence for multiple auditory areas. Neuroimage 6: 288-304. 
está generalmente bien desarrollada, presumiblemente por las aferencias provenientes del cuerpo geniculado medial del tálamo. La capa III está poblada por pequeñas y medianas células piramidales. Las células piramidales pequeñas de la capa III presentan un patrón de agrupación radial corto en columnas, con una parcial extensión a las capas corticales vecinas ${ }^{20}$.

En relación al análisis quimioarquitectónico de la corteza auditiva primaria, ésta se caracteriza por presentar una alta actividad de acetilcolinesterasa (AChE) y citocromooxidasa (CO). Específicamente, AChE, CO y parvoalbúmina presentan una alta expresión en la capa IV. Por otra parte, la mayor expresión de calretinina se presenta en la capa II y para calbindina se expresa con mayor intensidad en la capa $\mathrm{V}^{19}$. El análisis del tipo de receptor y su patrón de distribución permite obtener una asociación entre la función y los aspectos citoarquitectónicos de la organización de Al. Los principales receptores son los colinérgicos, muscarínicos (tipo M2) y nicotínicos. Estos receptores presentan un patrón de distribución con una alta expresión en las capas corticales medias de Al y una abrupta caída de su expresión en el borde de las áreas auditivas no primarias. Ya que los receptores colinérgicos están presentes en altos niveles en las capas que reciben información proveniente del tálamo (capas III/IV), se plantea que la corteza auditiva primaria tiene una fuerte modulación colinérgica18,21,22.

\section{Cortezas auditivas secundarias}

En el humano se han descrito siete áreas no primarias, PA (área auditiva posterior), LA (área auditiva lateral), LP (área auditiva lateroposterior), ALA (área auditiva anterolateral), AA (área auditiva anterior), MA (área auditiva medial) y STA (área

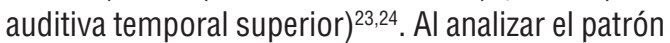
de tinción de CO y AchE se sugiere que existen 6 áreas no-primarias en un mismo nivel jerárquico de procesamiento (PA, LP, LA, ALA, AA, MA) y un área con un nivel de procesamiento superior (STA ${ }^{25}$.

El plano supratemporal está involucrado en el procesamiento de los estímulos acústicos para el reconocimiento y localización del sonido y la percepción del lenguaje. En particular en las áreas AA y ALA que han mostrado una activación selectiva para el reconocimiento del sonido, constituyendo la audición ventral 0 el "flujo del que"26, mientras que las áreas LA, PA y STA tienden a activarse ante la localización espacial del sonido y estarían más relacionadas al procesamiento de los ejes espaciales, formando el "flujo del donde" 0 audición dorsal ${ }^{27,28}$. Por otra parte, se sugiere que las áreas LA y STA tienen un rol importante en el análisis del lenguaje ${ }^{29}$, entrecruzando las vías de procesamiento ventral y dorsal, lo que sugiere que existe una vía dual para el procesamiento del lenguaje ${ }^{30}$. En la Figura 2 se muestra un esquema de este modelo del procesamiento auditivo central humano.

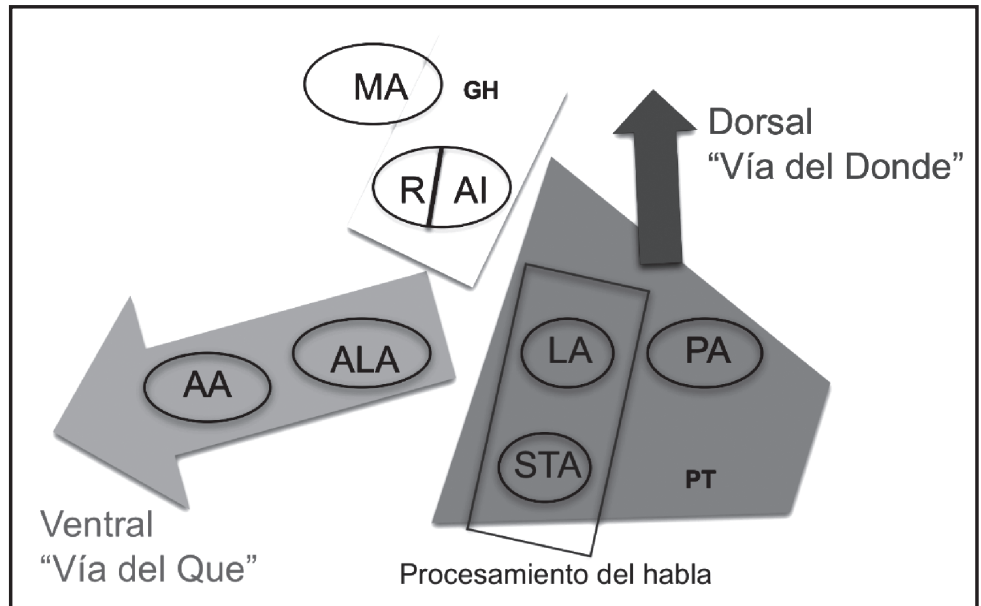

Figura 2. Esquema de la organización funcional de la corteza auditiva humana. Se muestran los diferentes niveles de procesamiento auditivo como en el plano temporal (PT) y el giro de Heschl (GH). Las diferentes áreas auditivas identificadas por medio de estudios histológicos y electrofisiológicos están divididas en áreas secundarias (AA, ALA, LA, PA, STA, MA) y áreas auditivas primarias $(\mathrm{R} / \mathrm{Al})$. Se sugiere que las áreas AA y ALA se activan selectivamente al reconocer sonidos, lo que se conoce como vía del "que", la cual está localizada ventralmente en el plano temporal. Mientras que las áreas LA, PA, y STA están localizadas por dorsal del plano temporal, se relacionan con la localización del sonido y es particular las áreas LA y STA con procesamiento del habla. 


\section{ASIMETRÍA ENTRE LOS HEMISFERIOS}

Se ha reportado en la literatura una asimetría entre los hemisferios cerebrales humanos, la que probablemente se debería a la especialización del hemisferio izquierdo para el lenguaje. Se describe que el giro de Heschl izquierdo presenta una ubicación más caudal que el derecho, a expensas de un crecimiento del giro temporal superior ${ }^{31}$. Esta es la principal diferencia morfológica del lóbulo temporal superior entre los humanos y los primates no humanos ${ }^{32}$.

En la misma línea, estudios realizados con resonancia nuclear magnética han evidenciado un mayor volumen de la región auditiva primaria izquierda en comparación a la derecha, y un mayor volumen de fibras corticales de conexión en la región izquier$\mathrm{da}^{33}$. Estos hallazgos podrían estar correlacionados con la dominancia del hemisferio izquierdo para el lenguaje y sugieren un rol de la corteza auditiva primaria izquierda en el procesamiento temporal de estímulos auditivos. La asimetría del giro de Heschl y del plano temporal estaría influenciada por factores genéticos y de plasticidad neuronal, ya que por ejemplo se ha descrito que pacientes con esquizofrenia presentan una reducción de la asimetría del plano temporal ${ }^{34}$, mientras que en músicos con oído absoluto (capacidad de identificar un tono musical) muestran un crecimiento del plano temporal en comparación a músicos sin esta condición ${ }^{35,36}$.

\section{FISIOLOGÍA DE LA CORTEZA AUDITIVA}

En la corteza auditiva se procesan los sonidos complejos, y a su vez ésta es necesaria para la construcción de la percepción del habla y de la música, junto con otras funciones complejas como son la localización del sonido e integración con otros sistemas sensoriales ${ }^{37}$. Estudios con resonancia magnética funcional han permitido determinar que la corteza auditiva primaria en seres humanos tiene al menos dos campos primarios paralelos con un orden tonotópico, conocidos como Al y $\mathrm{R}^{38}$. Estos campos estarían organizados de manera especular (ver Figura 1), y el borde que limita estas áreas corresponde a una zona de neuronas que responden preferencialmente a estímulos de frecuencias bajas ${ }^{39}$.
Una visión clásica de la función de la corteza auditiva plantea que la corteza auditiva primaria recibe exclusivamente información auditiva, mientras que progresivamente las cortezas secundarias mezclan la información auditiva con aquella de otros sistemas sensoriales, como visión u olfato ${ }^{40}$. Sin embargo, estudios recientes demuestran que la actividad de la corteza auditiva primaria puede ser modulada por otros sistemas sensoriales, como visual, somatosensorial y olfato ${ }^{41-44}$. Esto tiene implicancias terapéuticas en pacientes sordos, en los que se ha demostrado que la ausencia de estimulación auditiva provoca que la corteza auditiva responda a estímulos visuales ${ }^{45}$. De esta manera, una estimulación bimodal como visuo-auditiva 0 somato-auditiva podría tener mayor impacto al rehabilitar un paciente sordo que la estimulación unimodal ${ }^{46,47}$.

\section{EVALUACIÓN FUNCIONAL DE LA CORTEZA AUDITIVA}

Para evaluar la respuesta de la corteza a estímulos auditivos se utilizan diferentes tipos de potenciales auditivos como los potenciales evocados de latencia media 0 sensoriales, los potenciales de latencia tardía o cognitivos como los potenciales de disparidad o mismatch negativity ${ }^{48}$ y el p300 ${ }^{49}$. Si bien, los potenciales auditivos de tronco encefálico son los más utilizados en la práctica clínica, este examen sólo evalúa la vía auditiva central desde el nervio auditivo a los colículos inferiores ${ }^{50}$, por lo que muchas de las funciones de la corteza auditiva no son examinadas por este tipo de potencial. Los potenciales de disparidad o mismatch negativity se originan en la corteza auditiva primaria y no dependen de laatención del paciente para obtenerlos. Se utilizan para objetivar la discriminación auditiva, la memoria auditiva a corto plazo y patologías del lenguaje y neurológicas ${ }^{48}$.

Otro tipo de potencial auditivo de origen central son los potenciales de estado estable, cuya estructura generadora es mixta, involucrando vías de tronco encefálico y corteza auditiva ${ }^{51}$. Este tipo de potencial corresponde a una oscilación cerebral provocada por una amplitud modulada específica que lleva una frecuencia carrier ${ }^{52}$. Es conocido que la vía auditiva periférica y el tronco encefálico 
pueden seguir tasas de presentación de estímulos muy altas, superiores a los $80 \mathrm{~Hz}$, mientras que la corteza auditiva sólo puede seguir frecuencias menores a $80 \mathrm{~Hz}$, de esta manera, un potencial de estado estable provocado a altas tasas de presentación tendrá su origen principalmente en vías del tronco encefálico, mientras que uno provocado a bajas tasas, tendrá su origen principalmente en la corteza auditiva ${ }^{53}$.

Además de los potenciales de estado estable, existe otro tipo de oscilaciones cerebrales provocadas por estímulos auditivos, denominados los potenciales inducidos ${ }^{5,55}$. Este tipo de señal se obtiene al hacer un análisis frecuencial de la señal de la corteza auditiva, y se caracteriza por depender del tiempo en que la corteza auditiva ha estado en reposo por silencio. De esta manera, este potencial refleja la novedad de un estímulo auditivo, determinado por el largo del período de silencio previo a la estimulación. En resumen, existen múltiples tipos de potenciales evocados de corteza auditiva que detectan características del procesamiento auditivo, como la discriminación, atención y novedad de un estímulo auditivo, que en un futuro, al implementarlos en la práctica clínica permitirán mejorar la evaluación de un paciente con trastornos del procesamiento auditivo central.

\section{VÍAS DESCENDENTES DE LA CORTEZA AUDITIVA}

El sistema eferente auditivo está constituido por el sistema córtico-coclear y por el sistema olivococlear ${ }^{11}$. El sistema córtico-coclear es una compleja red neuronal que comienza en las neuronas piramidales de la capa V y VI de la corteza auditiva, luego proyecta por medio de dos vías principales, una hacia el cuerpo geniculado medial del tálamo y la otra al colículo inferior, complejo olivar superior y núcleos cocleares ${ }^{56,57}$. Posterior al trabajo de León y cols ${ }^{58}$ se postula que la vía eferente que va de la corteza a la cóclea tiene al menos dos vías funcionales que regulan la sensibilidad coclear probablemente en procesos que requieren de atención selectiva ${ }^{59}$ o el ciclo sueño vigilia ${ }^{60}$. Este tema ha sido recientemente revisado en un artículo de Terreros y cols (2013) que se recomienda leer a los interesados en el tema ${ }^{56}$.

\section{CONCLUSIONES}

La corteza auditiva es fundamental para la percepción y discriminación de estímulos auditivos complejos. Actualmente se postula que en la corteza auditiva primaria existen al menos dos campos con tonotopias especulares, mientras que las cortezas secundarias serían seis a siete campos que rodean a las cortezas primarias. El comprender su anatomía y fisiología es fundamental para el desarrollo de baterías diagnósticas y terapéuticas del procesamiento auditivo central.

\section{BIBLIOGRAFÍA}

1. WarRen R. Sound and the Auditory System. In: Auditory perception: A new analysis and synthesis. (Editor: Warren R). Cambridge, United Kingdom: The Press Syndicate of The University of Cambridge; 1999; 1-28.

2. Hudspeth AJ. Hearing In: Principles of Neuronal Science (eds. Kandel E, Jessell T, Schwartz J), Mc Graw- Hill. 2009; 592-613.

3. Délano PH. Central auditory system In: Atlas of otologic surgery and magic otology. (eds. De Souza C) Jaypee Brothers. 2011; 77-84.

4. Raphael Y, Altschuler RA. Structure and innervation of the cochlea. Brain Research Bulletin 2003; 60: 397-422.

5. Liberman MC. Single-neuron labeling in the cat auditory nerve. Science 1982; 216: 123941.

6. Wess I. Anatomía y Fisiología Coclear. En: Otoneurología clínica (ed Morales C). Editorial Universitaria; 1992; 13-26.

7. Malmierca MS, Izquierdo MA, Cristaudo S, Hernández 0, Pérez-González D, Covey E, Oliver DL. A discontinuous tonotopic organization in the inferior colliculus of the rat. J Neurosci2008; 28: 4767-76.

8. Molinari M, Dell'Anna ME, Rausell E, Leggio MG, HaShIKAWA T, JonES EG. Auditory thalamocortical pathways defined in monkeys by calcium-binding protein immunoreactivity. J Comp Neurol 1995; 362: 171-94.

9. Rakic P. Evolution of the neocortex: Perspective from developmental biology. Nature Reviews Neuroscience 2009; 10(10): 724-35. 
10. Amaral D. The Anatomical Organization of the Central Nervous System In: Principles of Neural Science (eds. Kandel E, Jessell T, Schwartz J), Mc Graw- Hill. 2000; 279-94.

11. Robles L, Délano PH. Efferent system. The senses: a comprehensive reference. 2008. London: Academic Press. 413-45.

12. BURTON H, Jones EG. The posterior thalamic region and its cortical projection in New World and Old World monkeys. The Journal of Comparative Neurology 1976; 168(2): 249301.

13. Hackett T, Preuss T, Kaas J. Architectonic identification of core region in auditory cortex of macaques, chimpanzees, and humans. The Journal of Comparative Neurology 2001; 1441: 197-222. Hackett T, Preuss T, KaAs J. Architectonic identification of core region in auditory cortex of macaques, chimpanzees, and humans. The Journal of Comparative Neurology 2001; 1441: 197-222.

14. OLRY R. Korbinian Brodmann (1868-1918). Journal of Neurology 2010; 257: 2112-3.

15. Brodmann K. (1909) Vergleichende Lokalisationslehre der Grosshirnrinde in ihren Prinzipien dargestellt auf Grund des Zellbaues. Johann Ambrosius Barth, Leipzig.

16. KAAS J, HACKETT T. Subdivisions of auditory cortex and processing streams in primates. Proceedings of the National Academy of Sciences 2000; 97: 11793-9.

17. Nieumenhuys R. Telencéfalo: neocorteza. En: El Sistema Nervioso Central Humano. (Eds: Nieuwenhuys R, Voogd J, Van Huijzen C). Editorial Médica Panamericana SA.; 2009; 491679.

18. Morosan P, Rademacher J, Palomero-Gallagher $\mathrm{N}$, Zilles K. Anatominal organization of the human auditory cortex: cytoarchitecture and transmitter receptors. In: The auditory cortex: a sintesis of human and animal research (Eds: König R, Heil P, Budinger E, Scheich H). Lawrence Erlbaum Associates; 2005; 27-50.

19. Clarke S, Morosan P. Architecture, connectivity, and transmitter receptors of human auditory cortex In: The Human Auditory Cortex (eds. Poeppel D, Overath T, Popper A, Fay R), Springer Handbook. 2012; 11-38.
20. Von Economo C, Koskinas GN. Die Cytoarchitectonik der Hirnrinde des erwachsenen menschen. 1925; Berlin: Julius Springer.

21. Zilles K, Palomero-Gallagher N, Grefkes C, Scheperuans F, Boy C, Amunts K et al. Architectonics of the human cerebral cortex and transmitter receptor fingerprints: Reconciling functional neuroanatomy and neurochemistry. European Neuropsychopharmacology 2002; 12: 587-99.

22. Zilles K, Schleicher A, Palomero-Gallagher N \& Amunts K. Quantitative analysis of cyto- and receptor architecture of the human brain. In J. C.Mazziotta \& A. Toga (Eds.), Brain mapping: The methods. Elsevier 2002; 573-602.

23. Rivier $F$ \& Clarke S. Cytochrome oxidase, acetylcholinesterase, and NADPH-diaphorase staining in human supratemporal and insular cortex: Evidence for multiple auditory areas. Neuroimagen 1997; 6: 288-304.

24. Wallace MN, Johnston PW \& Palmer AR. Histochemical identification of cortical areas in the auditory region of the human brain. Experimental Brain Research 2002; 143: 499-508.

25. Sacco CB, Tardif E, Genoud C, Probst A, Tolnay $M$, Janzer RC ET AL. GABA receptor subunits in human auditory cortex in normal and stroke cases. Acta Neurobiologiae Experimentalis (Warsaw) 2009; 69: 469-93.

26. Viceic D, Fornari E, Thiran JP, Maeder PP, Meuli R, AdrianI M et AL. Human auditory belt areas specialized in sound recognition: A functional magnetic resonance imaging study. Neuro Report 2006; 17: 1659-62.

27. GRIFFTHS TD \& WarRen JD. The planum temporale as a computational hub. Trends in Neurosciences 2002; 25: 348-53.

28. Van der Zwaag W, Gentile G, Gruetter R, Spierer $L$ \& ClaRKe $S$. Where sound position influences sound object representations: A 7T fMRI study. Neuroimagen 2011; 54: 1803-11.

29. ScotT SK \& JOHNSRUDE IS. The neuroanatomical and functional organization of speech perception. Trends in Neurosciences 2003; 26: 100-7.

30. HICKOK $G \&$ PoEPPEL D. The cortical organization of speech processing. Nature Reviews Neuroscience 2007; 8: 393-402.

31. KaAS JH, HACKETT T. The Functional Neuroanatomy of the Auditory Cortex. In: Allan I, Basbaum, Akimichi Kaneko, Gordon M. Shepherd and 
Gerald Westheimer, editors The Senses: A comprehensive Reference, Vol 3, Audition, Peter Dallos and Donata Oertel. San Diego: Academic Press; 2008; 765-780.

32. HACKETT T. Anatomical organization of the auditory cortex. Journal of the American Academy of Audiology 2008; 19: 774-9.

33. Penhune VB, Zatorre RJ, MacDonald JD, Evans AC. Interhemispheric anatomical differences in human primary auditory cortex: probabilistic mapping and volume measurements from magnetic resonance scans. Cerebral Cortex 1996; 6: 661-72.

34. Chance Sa, Casanova MF, Switala aE \& Crow TJ. Auditory cortex asymmetry, altered minicolumn spacing and absence of ageing effects in schizophrenia. Brain 2008; 131: 3178-92.

35. Schlaug G, Jancke L, Huang $Y$ \& Steinmetz $H$. In vivo evidence of structural brain asymmetry in musicians. Science 1995; 267: 699-701.

36. Wipe B, Kuroiwa M, Delano PH. Trastornos de la percepción musical. Revista de Otorrinolaringología y Cirugía de Cabeza y Cuello 2013; 73: 189-99.

37. Poeppel D, Overath T. Introduction: Why Human Auditory Cortex? In: The Human Auditory Cortex (eds. Poeppel D, Overath T, Popper A, Fay R), Springer Handbook. 2012; 11-38.

38. Formisano E, Kim DS, Di Salle F, van de Moortele PF, UGURBIL K, GoEBEL R. Mirror-symmetric tonotopic maps in human primary auditory cortex. Neuron 2003; 40: 859-69.

39. Saenz M, Langers DR. Tonotopic mapping of human auditory cortex. Hear Res 2014; 307: 42-52.

40. Mesulam MM. From sensation to cognition. Brain 1998; 121: 1013-52.

41. Brosch M, Selezneva E, Scheich H. Nonauditory events of a behavioral procedure activate auditory cortex of highly trained monkeys. $J$ Neurosci 2005; 25: 6797-806.

42. Délano PH, Elgueda D, Ramirez F, Robles L, Maldonado P. A visual cue modulates the firing rate and latency of auditory-cortex neurons in the Chinchilla. Journal of Physiology-Paris 2010; 104: 190-6.

43. Fu KM, Johnston TA, Shah AS, Arnold L, Smiley J, Hackett TA, Garraghty PE, Schroeder CE. Auditory cortical neurons respond to somatosensory stimulation. J Neurosci 2003; 23: 7510-5.

44. Cohen L, Rothschild G, Mizrahi A. Multisensory integration of natural odors and sounds in the auditory cortex. Neuron 2011; 72: 357-69.

45. Finney EM, Fine I, Dobkins KR. Visual stimuli activate auditory cortex in the deaf. Nat Neurosci 2001; 4: 1171-3.

46. Champoux F, Lepore F, Gagné JP, Théoret H. Visual stimuli can impair auditory processing in cochlear implant users. Neuropsychologia 2009; 47: 17-22.

47. Song JJ, Lee HJ, Kang H, Lee DS, Chang S0, Oh $\mathrm{SH}$. Effects of congruent and incongruent visual cues on speech perception and brain activity in cochlear implant users. Brain Struct Funct En prensa, 2014.

48. Carrasco L, Pavez E, Délano PH. Potencial de disparidad. Rev Otorrinolaringol Cir Cabeza Cuello 2008; 68: 185-92.

49. LINDEN DE. The p300: where in the brain is it produced and what does it tell us? Neuroscientist 2005; 11: 563-76.

50. Basterra J, Campos A. Exploración funcional del oído y de la audición En: Tratado de otorrinolaringología y patología cervicofacial. (eds Basterra J), Elsevier Masson. 2009; 4758.

51. Galambos R, Makeig S, Talmachoff PJ. A 40-Hz auditory potential recorded from the human scalp. Proc Natl Acad Sci USA 1981; 78: 26437.

52. Cabello P, Caro L. Audiometría de estado estable. Rev Otorrinolaringol Cir Cabeza Cuello 2007; 67: 162-6.

53. Picton TW, John MS, Dimitrijevic A, Purcell D. Human auditory steady-state responses. Int $J$ Audiol 2003; 42: 177-219.

54. Délano PH, Pavez E, Robles L. Stimulus-dependent oscillations and evoked potentials in Chinchilla auditory cortex. J Comp Physiol A 2008; 194: 693-700.

55. Dinse HR1, Krüger K, Akhavan AC, Spengler F, Schöner G, Schreiner CE. Low-frequency oscillations of visual, auditory and somatosensory cortical neurons evoked by sensory stimulation. Int J Psychophysiol 1997; 26: 205-27.

56. Terreros G, Wipe B, Leon A, Délano PH. Desde la corteza auditiva a la cóclea: Progresos en el 
sistema eferente auditivo. Rev Otorrinolaringol Cir Cabeza Cuello 2013; 73: 174-88.

57. Délano P, Robles I, Robles L. Sistema eferente auditivo. Rev Otorrinolaringol Cir Cabeza Cuello 2005; 65: 55-62.

58. León A, Elgueda D, Silva M, Hamamé C, Delano PH. Auditory cortex basal activity modulates cochlear responses in chinchillas. PlosOne 2012; 7(4): e36203.
59. Délano PH, Elgueda D, Hamame CM, Robles L. Selective attention to visual stimuli reduces coclear sensitivity in Chinchilla. Journal of Neuroscience 2007; 27: 4146-53.

60. Velluti R, Pedemonte M, García-Austt E. Correlative changes of auditory nerve and microphonic potentials throughout sleep. Hear Res 1989; 39: 203-8. 